\title{
SELF-NOISE IN INTERFEROMETERS
}

\author{
M. VIVEKANAND* \\ U.S.R.A., 600 Maryland Avenue, SW, suite 303, West Wing, Washington \\ DC 20024.
}

\author{
S. R. KULKARNI \\ Owens Valley Radio Observatory, 105-24, California Institute of \\ Technology, Pasadena, CA 91106.
}

\begin{abstract}
We study the distribution of noise in images synthesized by radio interferometers, focusing particularly on the noise due to source power fluctuations. For a total power interferometer, the r.m.s. fluctuation in the image consists of a term that is constant all over the map, which is the uncorrelated system noise divided by the number of telescopes in the interferometer, and a term that is the image itself. For a correlation interferometer the expression is not so simple but is qualitatively similar. Our results are consistent with intuitive expectations in various observing situations. We find similarities in selfnoise at optical and radio wavelengths.
\end{abstract}

\section{INTRODUCTION}

This article discusses the distribution of noise in images synthesized by interferometers operating mainly at radio wavelengths, although the formalism is basically wavelength independent. Noise usually implies fluctuations in the image due to random fluctuations at the output of receivers on account of the receiver noise, emission from the background such as the sky, the atmosphere and the ground, etc. Its distribution in the image has been well studied in the past (see Crane and Napier 1988, and references therein). In this article we focus special attention on the noise caused by fluctuations in the source power itself, the so called "self-noise". This problem has been studied independently by Anantharamiah et al. (1988) and Kulkarni (1989). The latter developed the analytic tools required for the study of this problem. We will use his results to derive an analytic expression for self-noise in terms of the image itself. At this conference we learnt that A.A. Deshpande (RRI, Bangalore) has obtained results similar to ours by an entirely independent approach.

Consider an interferometer consisting of $N$ telescopes. It would measure

* Naval Research Laboratory, Washington DC; Visiting Associate at California Institute of Technology, Pasadena; On leave from Raman Research Institute, Bangalore. 
instantaneously $N_{b}=N(N-1) / 2$ visibilities R and $N$ total powers z. Using the Van-Cittert Zernike theorem the "dirty image" $I$ is obtained by Fourier transforming the visibilities.

$$
I(\theta)=\frac{1}{N^{2}}\left[\sum_{k=1}^{N} \mathbf{z}_{k}+\sum_{l=1}^{N_{b}} \mathrm{R}_{l} \exp -j\left[\mathbf{w}_{l} \theta\right]+\mathrm{R}_{l}^{*} \exp +j\left[\mathbf{w}_{l} \theta\right]\right]
$$

where $\theta$ is the coordinate in the image plane (a one-dimensional image will be adequate for our purpose) and $w$ is the spatial frequency of the relevent baseline* $e^{*}$ The usual practice in radio synthesis imaging is not to include the total powers and to normalize the right hand side of eq. (1) by $2 N_{b}$ instead of $N^{2}$. We will adopt the above formalism as it yields an analytic expression for self-noise; but the qualitative behaviour of self-noise is independent of whether all or some or even none of the total powers are included. Also, the visibilities are usually weighted before Fourier inversion, but we will neglect this as it will not alter our results which are stated in terms of the dirty image. Finally we will ignore deconvolution processes such as CLEAN and MEM that are not expected to modify fundamentally the noise in the image.

The noise in the image can be defined in terms of its variance

$$
\begin{aligned}
& \sigma^{2}(\theta)=\left\langle I^{2}(\theta)\right\rangle-\langle I(\theta)\rangle^{2}=\frac{1}{N^{4}}\left[\sum_{k=1}^{N} \sum_{l=1}^{N} \operatorname{COV}\left[\mathbf{z}_{k}, \mathbf{z}_{l}\right]\right. \\
& +2 \sum_{k=1}^{N} \sum_{l=1}^{N_{b}} \operatorname{COV}\left[\mathrm{z}_{k}, \mathrm{R}_{l}\right] \exp -j\left[\mathrm{\omega}_{l} \theta\right]+\operatorname{COV}\left[\mathrm{z}_{k}, \mathrm{R}_{l}^{*}\right] \exp +j\left[\mathrm{\omega}_{l} \theta\right] \\
& +\sum_{k=1}^{N_{b}} \sum_{l=1}^{N_{b}} \operatorname{COV}\left[\mathbf{R}_{k}, \mathbf{R}_{l}\right] \exp -j\left[\left(\mathbf{w}_{k}+\mathbf{w}_{l}\right) \theta\right]+\operatorname{COV}\left[\mathbf{R}_{k}, \mathbf{R}_{l}^{*}\right] \exp -j\left[\left(\mathbf{w}_{k}-\mathbf{w}_{l}\right) \theta\right] \\
& \left.+\operatorname{COV}\left[\mathrm{R}_{k}^{*}, \mathrm{R}_{l}\right] \exp +j\left[\left(\mathrm{~W}_{k}-\mathrm{W}_{l}\right) \theta\right]+\operatorname{COV}\left[\mathrm{R}_{k}^{*}, \mathrm{R}_{l}^{*}\right] \exp +j\left[\left(\mathrm{~W}_{k}+\mathrm{W}_{l}\right) \theta\right]\right](2)
\end{aligned}
$$

where the angle brackets refer to ensemble averages, and $C O V[x, y]$ is the covariance of the quantities $x$ and $y$, defined as $\langle x y\rangle-\langle x\rangle\langle y\rangle$. Thus the noise $\sigma(\theta)$ at any point $\theta$ in the image depends upon the covariances between the ensemble of visibilities $R$ and total powers $Z$. The signal-to-noise ratio in the image $(S N R)$ is given by $I(\theta) / \sigma(\theta)$.

\section{NOISE IN SYNTHESIZED IMAGE}

Following Kulkarni (1989) it can be shown that

$$
\begin{aligned}
& \operatorname{COV}\left[\mathrm{R}_{u v}, \mathrm{R}_{u v}^{*}\right]=\frac{1}{M}[S+N]^{2} \quad \operatorname{COV}\left[\mathbf{R}_{u v}, \mathbf{R}_{u v}\right]=\frac{1}{M}\left[\mathbf{I}_{u v} \mathbf{r}_{u v}\right] \\
& \operatorname{COV}\left[\mathbf{R}_{u v}, \mathbf{R}_{u w}^{*}\right]=\frac{1}{M}[S+N] \mathbf{r}_{v w}^{*} \quad \operatorname{COV}\left[\mathbf{R}_{u v}, \mathbf{R}_{u w}\right]=\frac{1}{M}\left[\boldsymbol{r}_{u v} \boldsymbol{I}_{u w}\right] \\
& \operatorname{COV}\left[\mathrm{R}_{s t}, \mathrm{R}_{u v}^{*}\right]=\frac{1}{M}\left[\mathrm{r}_{s u} \mathrm{r}_{v t}\right] \quad \operatorname{COV}\left[\mathrm{R}_{s t}, \mathrm{R}_{u v}\right]=\frac{1}{M}\left[\mathrm{r}_{s v} \mathrm{r}_{u t}\right] \\
& \operatorname{COV}\left[\mathrm{z}_{u}, \mathrm{z}_{u}\right]=\frac{1}{M}[S+N]^{2} \quad \operatorname{COV}\left[\mathrm{z}_{u}, \mathbf{z}_{v}\right]=\frac{1}{M}\left[\mathrm{r}_{u v} \mathrm{r}_{u v}^{*}\right] \\
& \operatorname{COV}\left[\mathbf{z}_{u}, \mathbf{R}_{u v}\right]=\frac{1}{M}[S+N] \mathbf{r}_{u v} \quad \operatorname{COV}\left[\mathbf{z}_{u}, \mathbf{R}_{s t}\right]=\frac{1}{M}\left[\mathbf{r}_{u s}^{*} \mathbf{r}_{u t}\right]
\end{aligned}
$$

* $\mathrm{R}$ will have either two subscripts referring to the two telescopes involved, or a single subscript referring to the baseline involved, depending upon the convenience. 
where $S$ and $N$ are the true source and noise powers respectively at any telescope, and $\boldsymbol{r}_{u v}$ is the true visibility on the baseline formed by telescopes $u$ and $v$. One notices that the covariance is more significant among visibilities that have more telescopes in common.

Substituting the above covariances into eq. (2) it can be shown that

$$
\begin{aligned}
\sigma(\theta) & =\frac{1}{\sqrt{M} N^{2}}\left[N(S+N)+\sum_{k=1}^{N_{b}} r_{k} \exp -j\left[w_{k} \theta\right]+r_{k}^{*} \exp +j\left[w_{k} \theta\right]\right] \\
& =\frac{1}{\sqrt{M}}\left[\frac{N}{N}+I(\theta)\right]
\end{aligned}
$$

$\sigma(\theta)$ consists of a uniform value of $N / N$ at all $\theta$ plus a term $I(\theta)=\frac{1}{N^{2}}[N S+$ $\left.\sum_{k=1}^{N_{b}} \mathbf{r}_{k} \exp -j\left[w_{k} \theta\right]+r_{k}^{*} \exp +j\left[W_{k} \theta\right]\right]$ equal to the dirty image $I(\theta)$ without the noise $N$, both divided by $\sqrt{M}$. For a correlation interferometer the expression for noise $\sigma_{c}$ is

$$
\sigma_{c}(\theta)=\frac{1}{\sqrt{M} 2 N_{b}} \sqrt{2 N_{b}(S+N)^{2}+2(N-2)(S+N) 2 N_{b} i(\theta)+\left.4 N_{b}^{2} i^{2}(\theta)\right|_{*}}
$$

where $i(\theta)=\frac{1}{2 N_{b}} \sum_{k=1}^{N_{b}} \mathbf{r}_{k} \exp -j\left[\mathrm{w}_{k} \theta\right]+\mathbf{r}_{k}^{*} \exp +j\left[\mathrm{w}_{k} \theta\right] \approx I(\theta)-S / N$ is the dirty image $I(\theta)$ without the total powers $z$ (this is the result from a correlation interferometer), and " $\left.i^{2}(\theta)\right|_{*}$ " is the square of $i(\theta)$ with some side-bands missing in the frequency domain. The missing sidebands correspond to products of visibilities that have one telescope in common. Equation 5 is almost the same as eq. (4), but for the fact that the square can not be completed, and that the square of the image has some side-bands missing. For a point source at the phase center eq. (5) simplifies to

$$
\sigma_{c}(\theta)=\frac{1}{\sqrt{M}} \sqrt{S^{2}+\frac{2 S N}{N}+\frac{N^{2}}{N(N-1)}}
$$

For a two element interferometer eq. (6) reduces to the familiar formula of Crane and Napier (1988).

\section{DISCUSSION}

Consider first the case when the source power $S$ is much smaller than the noise power $N$. Then $I(\theta)$ can be ignored in eq. (4) and the noise in the image is given by $N / \sqrt{M} N$, and the $S N R$ is given by $I(\theta) \sqrt{M} N / N$. The $S N R$ in the image is inversely proportional to the noise power $N$, directly proportional to the square root of the duration of observation, and directly proportional to the number of telescopes $N$ in the interferometer. It is also directly proportional to the collecting area of each telescope due to $I(\theta)$. This is a very familiar result, except that usually the total powers are not included in the analysis, which alters the $N$ above to $\sqrt{N_{b}}$ (Crane and Napier 1988).

Next consider a strong unresolved source. The image $I(\theta)$ reduces to the point source flux $S$ at one point in the image, and $N$ can be ignored in eq. 
(4). Then the noise in the image is given by $S / \sqrt{M}$ and the $S N R$ is merely $\sqrt{M}$, independent of the collecting area of each telescope or the number of telescopes $N$. This intuitive result is also well known.

When the strong source in resolved, $I(\theta)$ reduces to $S / N$ as the summation over the visibilities in eq. (1) goes to zero. Thus for a strong resolved source, the noise in the image is $\sigma(\theta)=(S+N) / \sqrt{M} N$. The source power fluctuations contribute like uncorrelated receiver noise power because the source completely fills the interferometer's beam. This result too has been noted by Crane and Napier (1988).

What we have derived so far is strictly valid for a snap-shot mode of observation, in which all visibilities are measured simultaneously. What happens when the $(U, V)$ plane is synthesized slowly by earth rotation, using a smaller number of telescopes? For proper comparison consider two interferometers, one consisting of $m$ telescopes, and the other consisting of $N$ telescopes. Instantaneously the former measures $m_{b}=m(m-1) / 2$ visibilities, and has to observe for say $G$ sessions to measure the $N_{b}$ visibilities that the latter interferometer measures instantaneously $\left(N_{b}=G m_{b}\right)$. The noise in a slow-synthesis image can be shown to be

$$
\sigma^{\prime}(\theta) \approx \frac{1}{\sqrt{M}} \sqrt{\left[\frac{N}{N}+\frac{\left\langle I_{k}(\theta)\right\rangle}{\sqrt{G}}\right]^{2}+\frac{\left\langle I_{k}^{2}(\theta)\right\rangle-\left\langle I_{k}(\theta)\right\rangle^{2}}{G}}
$$

which is true for large $m$ and $N .\left\langle I_{k}(\theta)\right\rangle$ is the average of the $G$ individual dirty images without noise, while $\left\langle I_{k}^{2}(\theta)\right\rangle$ is the average of the square of these dirty images. Equation (7) implies that self-noise in a slowly synthesized image depends upon an average dirty beam that in general has no resemblance to the final dirty beam of the interferometer. So self-noise may be found in those regions of the map where no source exists at all! To compare further consider an image that consists of a strong point source at the phase center of the interferometer. Then $\sigma^{2} \approx S^{2} / M G$ while $\sigma^{2}=S^{2} / M$. The ratio of the two variances is inversely proportional to the number of sessions $G$, which is intuitively reasonable. Now $\sqrt{G}$ can be a large number, of the order of $\approx 25-50$, particularly in VLBI where a few telescopes are used to synthesize for long times. Thus self noise in a slowly synthesized image qualitatively varies directly with the rate at which the image is synthesized.

Prasad and Kulkarni (1989) have obtained an analytic solution to selfnoise at optical wavelength. For a total power interferometer our solution is similar to theirs; for a correlation interferometer the self-noise at optical wavelengths depends upon the beam combination geometry, unlike the radio case.

\section{REFERENCES}

Anantharamiah, K.R., Ekers, R.D., Radhakrishnan, V., Cornwell, T.J. and Goss, W.M., 1988, in Synthesis Imaging In Radio Astronomy, ed.

R.A.Perley, F.R.Schwab and A.H.Bridle, Pub. A.S.P., $\underline{6}$, p. 431.

Crane, P.C. and Napier, P.J., 1988, in Synthesis Imaging In Radio Astronomy, ed. R.A.Perley, F.R.Schwab and A.H.Bridle, Pub. A.S.P., $\underline{6}$, p. 139.

Kulkarni S.R., 1989, Astron. J., 98, p. 1112.

Prasad, S. and Kulkarni, S.R., 1989, J. Opt. Soc. Am. A, ㅁ, p. 1702. 
J. Delannoy: What is the practical value of "the number of sessions"? Vivekanand: It is "The number of telescopes"

R. Ekers: Although your formalism has totally obscured the physics it may be useful to address some other cases similar to the self-noise case: 1) fluctuations in atmospheric attenuation; 2) phase fluctuations (e.g. due to the atmosphere). Note that this is not like the vector noise case and may be related to your treatment.

Vivekanand: I can not agree that our simple formula for self-noise actually obscures the physics, instead of clarifying it. The derivation is certainly difficult, since we start from first principles, but one is welcome to disregard our derivation and consider merely the final formula for self-noise. We have in a manner considered phase fluctuations due to the atmosphere, in that we derived self noise in images that are made from bispectra. We will certainly consider the other aspects, and thank you for mentioning them. 